\title{
Nuclear position and shape deformation of chromosome 8 territories in pancreatic ductal adenocarcinoma
}

\author{
Sylvia Timme ${ }^{\mathrm{a}}$, Eberhard Schmitt ${ }^{\mathrm{b}}$, Stefan Stein ${ }^{\mathrm{b}}$, Jutta Schwarz-Finsterle ${ }^{\mathrm{b}}$, Jenny Wagner ${ }^{\mathrm{b}}$, \\ Axel Walch ${ }^{\mathrm{c}}$, Martin Werner ${ }^{\mathrm{a}}$, Michael Hausmann $^{\mathrm{b}}$ and Thorsten Wiech ${ }^{\mathrm{a}, \mathrm{d} *}$ \\ ${ }^{a}$ Institute of Pathology, University Hospital Freiburg, Freiburg im Breisgau, Germany \\ ${ }^{\mathrm{b}}$ Kirchhoff Institute for Physics, University of Heidelberg, Heidelberg, Germany \\ ${ }^{\mathrm{c}}$ Helmholtz Zentrum München, German Research Center for Environmental Health, Institute of Pathology, \\ Neuherberg, Germany \\ ${ }^{\mathrm{d}}$ Centre of Chronic Immunodeficiency (CCI), University Medical Center Freiburg and University of Freiburg, \\ Freiburg im Breisgau, Germany
}

\begin{abstract}
Cell type specific radial positioning of chromosome territories (CTs) and their sub-domains in the interphase seem to have functional relevance in non-neoplastic human nuclei, while much less is known about nuclear architecture in carcinoma cells and its development during tumor progression. We analyzed the 3D-architecture of the chromosome 8 territory (CT8) in carcinoma and corresponding non-neoplastic ductal pancreatic epithelium. Fluorescence-in-situ-hybridization (FISH) with whole chromosome painting (WCP) probes on sections from formalin-fixed, paraffin wax-embedded tissues from six patients with ductal adenocarcinoma of the pancreas was used. Radial positions and shape parameters of CT8 were analyzed by 3Dmicroscopy. None of the parameters showed significant inter-individual changes. CT8 was localized in the nuclear periphery in carcinoma cells and normal ductal epithelial cells. Normalized volume and surface of CT8 did not differ significantly. In contrast, the normalized roundness was significantly lower in carcinoma cells, implying an elongation of neoplastic cell nuclei. Unexpectedly, radial positioning of CT8, a dominant parameter of nuclear architecture, did not change significantly when comparing neoplastic with non-neoplastic cells. A significant deformation of CT8, however, accompanies nuclear atypia of carcinoma cells. This decreased roundness of CTs may reflect the genomic and transcriptional alterations in carcinoma.
\end{abstract}

Keywords: Pancreatic adenocarcinoma, nuclear architecture, chromosome 8 territory, radial positioning, shape of chromosome 8 territory

\section{Introduction}

Understanding the nuclear architecture [6,9] is indispensable for understanding nuclear functions and dysfunctions during cellular changes in neoplastic

*Corresponding author: Dr. Thorsten Wiech, Institute of Pathology, University Hospital Freiburg, Breisacher Strasse 115a, 79106, Freiburg im Breisgau, Germany. Tel.: +49 761270 8119; Fax: +49 761270 8004; E-mail: thorsten.wiech@uniklinik-freiburg.de. transformation. At present we only know little about the range and dynamic changes of the cell type specific nuclear architecture that can occur during cell cycle, terminal cell differentiation and abnormal cellular development [2, 16, 19, 23, 32, 33]. Moreover, we often disregard the interplay between 3D nuclear architecture and function $[8,11]$.

Current knowledge about nuclear architecture and spatial chromatin organization acknowledges that individual chromosomes in the cell nucleus occupy distinct chromosome territories (CTs) divided into function- 
ally determined sub-chromosomal domains [7, 8, 12]. The term "chromosome territory" was first introduced by Boveri [4]. The positioning of the CTs and their sub-chromosomal domains in the interphase nucleus is non-random and well organized $[3,12,20,23,26$, $30,35]$. One of the main features describing cell type specific arrangements of CTs is the relative radial position, i.e. the relative radial distance of the CT to the center of the nucleus. CT arrangements seem to be mostly associated with the gene density and gene activation of the respective chromosome [42]. For example chromosomes with a low gene density, such as chromosome 18 , tend to be located more peripheral than chromosomes with a higher gene density, such as chromosome 19 [5, 10, 13, 23, 35]. Radial positioning of individual CTs seems to be unique in different tissues [31] and it has been described to be evolutionarily conserved, for instance, in lymphocytes and fibroblasts over 300 million years [43, 44]. These facts suggest a functional relevance of non-random CT positioning $[14,23,40]$. Changes of spatial organization are associated with changes of gene expression and genomic and functional alterations during neoplastic transformation as it has been reported for chromosome 18 and the gene BCL2 [46]. In breast cancer cells distinct positional changes of several gene domains were noticed during malignant transformation [28].

Using fluorescence in situ hybridization (FISH) with whole chromosome painting (WCP) probes on sections from formalin-fixed, paraffin wax-embedded human tissues we showed that it is possible to study genome architecture of individuals in the natural histological context $[45,46]$. In comparison to commonly applied cell cultures, the methodical advantage of this approach is the preservation of the orientation and position of the nuclei within the tissue, as well as their spatial relation to supranuclear or extracellular structures like basement membranes or ductal lumina.

Here, we analyzed the relative radial positioning and the shape parameters volume, surface, and roundness of the chromosome 8 territory (CT8) in non-neoplastic human pancreatic ductal epithelium in comparison to pancreatic ductal adenocarcinoma as a model of a malignant transformation. Since genomic alterations of chromosome 8, such as amplification of CMYC [36] are frequent in pancreatic ductal adenocarcinoma, we expected changes of the CT8 architecture.

Previous investigations indicated a slight shift towards the nuclear periphery and a reduced roundness of CT8 in one case of ductal adenocarcinoma of the pancreas compared to corresponding normal epithelium [45]. In order to investigate whether this was an accidental observation or reflects a recurrent alteration, further individuals were analyzed in this study. Architectural parameters of CT8 were evaluated in additional cases of pancreatic ductal adenocarcinoma in comparison to corresponding normal ductal epithelium.

\section{Materials and methods}

\subsection{Tissues}

In this study tissue samples from pancreatectomy specimens of 6 patients with ductal adenocarcinoma of the pancreas (according to the WHO-classification [17]) were analyzed (see also case description in Section 3). The material was fixed in buffered formaldehyde $(4 \%$ in phosphate-buffered saline (PBS)) and paraffin wax-embedded. Histologically normal ductal epithelium and corresponding invasive ductal adenocarcinoma of the pancreas were selected mostly from the same patient (Fig. 1). Grading categories G1-G3 [17] and tumor stages pT2 and pT3 according to the UICC (Union Internationale Contre le Cancer) [39] were represented.

\subsection{Tissue microarrays}

Tissue microarrays (TMA) were assembled from selected areas of non-neoplastic pancreatic tissue including ducts without dysplasia or inflammation (normal) and areas of ductal adenocarcinoma (carcinoma). 2-mm tissue-cores were taken from the paraffin blocks (donator-block) and transferred into a new paraffin block (acceptor block), creating a TMA-block including 12 tissue cores from all patients. For hematoxylin and eosin staining, H\&E staining (Fig. 1A, B) and FISH analysis (Fig. 1C, D) sections from the TMA were prepared and mounted on glass slides. The first and the last section $(2 \mu \mathrm{m})$ were stained with hematoxylin and eosin for morphological control, the section in between $(15 \mu \mathrm{m})$ was used for FISH.

\subsection{DNA probe set}

A commercially available fluorescence-labeled whole chromosome painting (WCP) DNA probe for chromosome 8 labeled with spectrum green (Abbott 

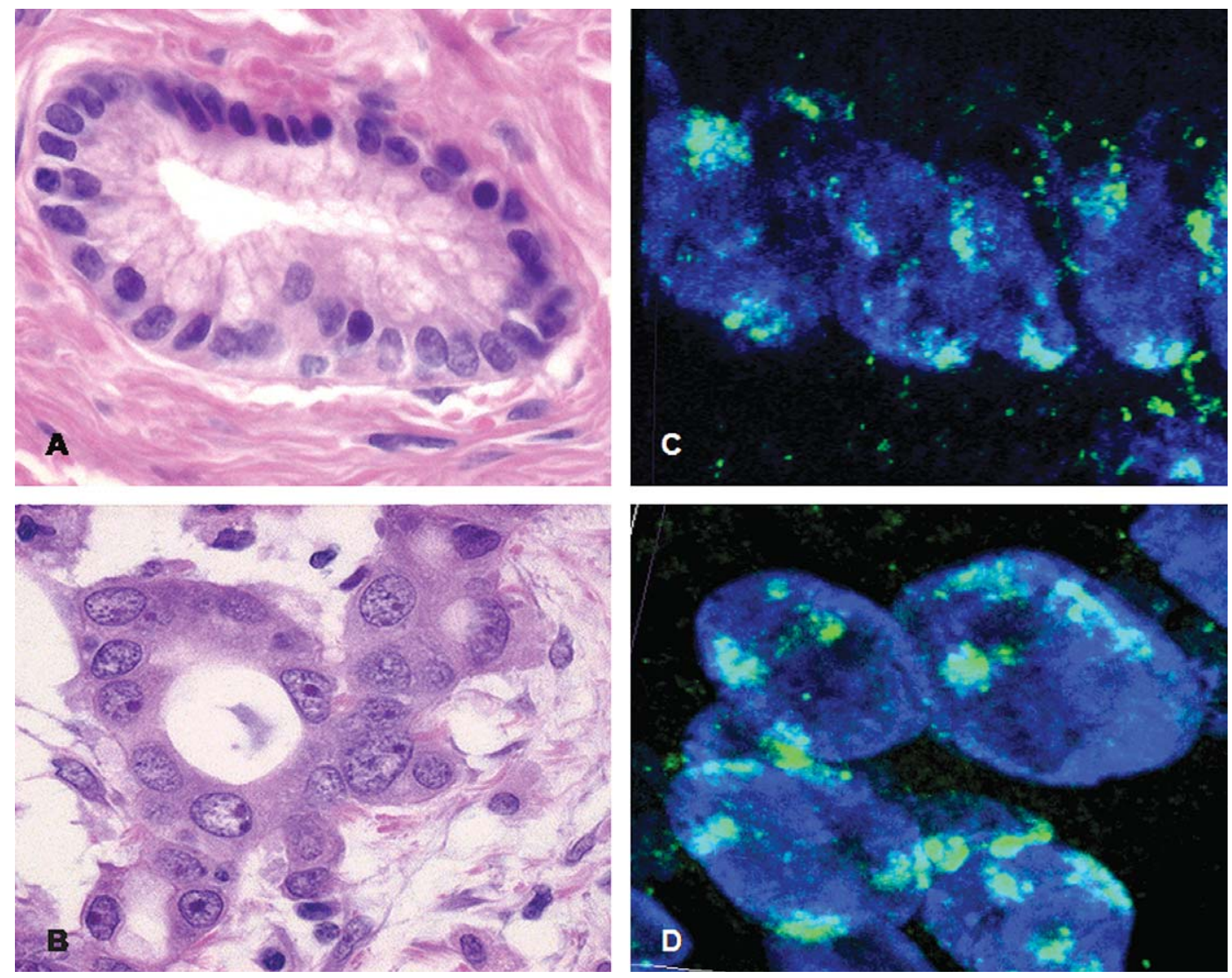

Fig. 1. A: Non-neoplastic (normal) pancreatic ductal epithelium: H\&E staining, 600×; B: Neoplastic epithelium (pancreatic ductal adenocarcinoma): H\&E staining, 600×; C: Non-neoplastic (normal) pancreatic ductal epithelium: WCP8 FISH, 1000×; D: Neoplastic epithelium (pancreatic ductal adenocarcinoma): WCP8 FISH, 1000×.

Molecular Diagnostics, Illinois, USA) was applied to the sections according to the manufacturer's instructions.

\subsection{Fluorescence in situ Hybridization (FISH)}

Paraffin was removed by xylene for $30 \mathrm{~min}$ and isopropanol for $3 \mathrm{~min}$. The slides were hydrated over a graded series of ethanol (100\%, 96\%, $70 \%$ and $50 \%)$ and PBS ( $\mathrm{pH} 7$ ). The tissue was pre-treated by heating the slides for $20 \mathrm{~min}$ in a microwave oven $(180 \mathrm{~W})$ in citrate buffer $(\mathrm{pH} 7)$ followed by pronase $\mathrm{E}(0.05 \%)$ digestion for $3 \mathrm{~min}$ at $37^{\circ} \mathrm{C}$. After washing three times for $3 \mathrm{~min}$ with PBS ( $\mathrm{pH} 7$ ), the slides were denatured in $70 \%$ formamide and $2 \times$ standard saline citrate (SSC), $\mathrm{pH} 7$, for $15 \mathrm{~min}$ at $73^{\circ} \mathrm{C}$. Then the slides were dehydrated over a graded series of ethanol $(70 \%$, $90 \%, 100 \%$, at $-20^{\circ} \mathrm{C}$ ) and air dried. The fluorescence- labeled DNA probes were added to the pre-treated slides according to the manufacturer's instructions and incubated for $48 \mathrm{~h}$ at $37^{\circ} \mathrm{C}$. After in situ hybridization, the slides were placed in $2 \times \mathrm{SSC} / 0.1 \% \mathrm{NP}-40$ (pH 7.4) at $73^{\circ} \mathrm{C}$ for 2 min, embedded with an antifade solution (Vectashield, Butlingame, USA) after incubating with $4^{\prime}, 6$-diamino-phenylindole (DAPI) as nuclear counterstain and covered with a standard coverglass.

\subsection{Fluorescence microscopy and 3D image acquisition}

Three dimensional image stacks (voxel size $102 \times 102 \times 325 \mu^{3}$ ) of the FISH-labeled chromosome 8 and the counterstained nuclei were acquired with a Zeiss Axioplan2 imaging microscope (Carl Zeiss Jena, Jena, Germany). The microscope was equipped with a PlanApochromat 100x/NA1.4 oil 
objective lens, filters for DAPI and SpectrumGreen (FITC) illumination and an ApoTome (Carl Zeiss Jena, Germany) for optical sectioning through the specimens. The setup was controlled by AxioVision software that also managed the image acquisition by an AxioCam b/w CCD camera (Carl Zeiss Jena, Germany). The image stacks were recorded sequentially in the two color planes (green for SpectrumGreen; blue for DAPI) and exported as uncompressed 8-bit TIFF files to the image processing software ImageJ.

\subsection{Quantitative geometric evaluation of images}

Radial positioning, volume, surface, and roundness of CT8 were processed by an image analyzing software package using a voxel-based algorithm. The evaluation included 20 to 40 CT8 of each specimen of both normal and carcinoma from 4 (normal) and 5 (carcinoma) patients and 1 test specimen, respectively.

Using the image processing program ImageJ, the cell nuclei were interactively segmented and optically isolated from the surrounding tissue by visual inspection. This pre-segmentation step helped to avoid misinterpretation due to overlapping or closely attached cell nuclei in the tissue sections. The preprocessed TIFF images were converted into KDF format for further evaluation using program packages running under Linux 8.0 (SuSE Linux) on Grid connected computing clusters as described in detail elsewhere [37, 38].

In a first image processing step after presegmentation, non-specific artifacts, staining background and detection noise were reduced applying a background subtraction and a weak Gaussian filter. Processing the image stacks, the exact border of the nucleus and the center of gravity were automatically determined using the 3D image stack of the DAPIcounterstain. From the other color channel (green), the CT8 territory was determined automatically in an analogous way.

The parameters volume, surface, and roundness were determined by a software package that quantifies geometric data over a reasonable threshold spectrum, here for intensities between 0 and 255 [37, 38, 41]. Volume, surface, and roundness were calculated independently from the voxel intensity for each value within the threshold spectrum chosen, in our analysis between threshold values 100 and 250. By this means it was also possible to analyze CTs which were not uniformly, i.e. fragmentedly stained due to pretreatment procedures applied for WCP. Volumes of irregular shapes were obtained by voxel counting of the connected voxels above the given intensity threshold. Thus, for a specific threshold value, all voxels belonging to the territory under consideration defined by the segmentation process with an intensity value at least as high as the threshold are attributed to the territory volume. Intensity values below the threshold are ignored. The volume of the territory is the sum of the volumes of those voxels whose intensity value is above the threshold. Let $\mathrm{N}$ be the number of these beforehand mentioned voxels, then the territory volume $\mathrm{V}$ is given by

$$
\mathrm{V}=\mathrm{N} \mathrm{dx^{2 } \mathrm { dz }}
$$

( $d x$ the lateral voxel size and $d z$ the axial voxel size). In relation to the total voxel number it defines the volume of the territory for the respective threshold. These values are normalized with respect to the maximal value found, in our case for each patient and tissue type the volume value for threshold 100 .

In an analogous way, by considering the bounding surfaces of voxels belonging to the surface of the territory, the threshold dependent surface values are determined and normalized accordingly. In that case, for the bounding face the specific area has to be taken into account:

$$
\mathrm{S}=\mathrm{N}_{\mathrm{LBF}} \mathrm{dx} \mathrm{x}^{2}+\mathrm{N}_{\mathrm{VBF}} \mathrm{dxdz}
$$

where $\mathrm{N}_{\mathrm{LBF}}$ denotes the number of all lateral bounding faces and $\mathrm{N}_{\mathrm{VBF}}$ analogously the number of all vertical bounding faces of those voxels which belong to the volume of the territory but face the outer region.

The roundness value $\mathrm{R}$ is defined as the normalizing quotient of respective powers of territory volume $\mathrm{V}$ and surface $\mathrm{S}$ :

$$
\mathrm{R}=36 \pi \cdot \mathrm{V}^{2} / \mathrm{S}^{3}
$$

Note that $0 \leq R \leq 1$ and $R=1$ for a sphere.

To compute the radial distribution of a territory, each point on the segmented nuclear border was normalized to the relative radius $r_{0}=100 \%$ to enable comparing nuclei of different shapes and sizes. In the same way, the segmentation of the labeled CT8 was performed in each 3D data stack. The segmented nuclear space was divided into 25 equidistant shells with a thickness of $\Delta r=r_{0} / 25$. For each of these shells, all voxels assigned to a given labeled genome region were iden- 
tified and their fluorescence intensities were summed. At the end, the sum of all voxel intensities measured in all nuclei was set to $100 \%$ for each color channel. With this normalization, the radial positioning distribution was determined.

For visual display of the curves based on the data, we did not connect the data points (shown separately) directly, but used interpolating splines [34] to reduce the statistical noise in order to point out the general characteristics of the functions.

\subsection{Statistical analysis}

To evaluate and compare data of different tissues and to show statistically significant differences, two strategies were applied. For volume, surface, and roundness values, the threshold curves could be compared. The invariance with respect to threshold shifts was taken into account by the normalizing step. Therefore, we applied the standard Mann-Whitney-U-Test [1], an alternative to the $t$-test for independent groups, to compare the values of two different tissues in dependence of the threshold. In the case of roundness values, we chose a limited range of threshold values, where the function showed a linear behavior. For this range, a linear fit was also calculated. Typically, the number of points between the lower and upper linear fit threshold (LFT) was 12 to 15.

Radial positioning distributions were tested by the double-sided Kolmogorov-Smirnov (KS)-Test [21] to ensure that two data sets were significantly different. We decided to use this non-parametric test as there were no indications for normal distributions of the concerning measurements. This test is known to be stable and sensitive to differences.

\section{Results}

Our study is based on the analysis of the cell nuclei of 4 patients (patients $1-4$ in the respective figures) with both, non-neoplastic (normal) and neoplastic (carcinoma) tissue sections, and one patient with carcinoma tissue only (patient 5 in the respective figures). In the tables, the normal tissues (patients 1-4) carry numbers 1n through $4 \mathrm{n}$, the corresponding carcinoma tissues (patients 1-4) numbers 1c through 4c. The single carcinoma tissue is number $5 \mathrm{c}$. In addition, to validate the methods applied, we analyzed several tissue sections of a further "test" patient independently in parallel comparing the resulting data (here exemplifying numbers 6 and 7).

Figure 1 shows a typical example of a non-neoplastic (normal) and neoplastic (carcinoma) tissue, respectively. All 3D images of cell nuclei and hybridized CT8 were quantitatively analyzed in terms of their 3D radial positioning and geometric parameters, such as territory volume, surface, and roundness. In addition, we also computed cumulative roundness curves and cumulative radial distributions for the normal group $1 \mathrm{n}$ through $4 \mathrm{n}$ (number $8 \mathrm{n}$ in the tables) and for the carcinoma group $1 \mathrm{c}$ through $5 \mathrm{c}$ (number $9 \mathrm{c}$ in the tables).

\subsection{Radial positioning of CT 8}

In non-neoplastic epithelium from four patients (normal group), the average radial position of CT8 was in the nuclear periphery at approximately $75 \%$ of the nuclear radius (Fig. 2A). The interpolating spline curves do not deviate very much from the measurement points and have their maximum between $65 \%$ and $78 \%$ of the nuclear radius at values between $9.5 \%$ and $12 \%$ of relative DNA-content within the respective nuclear shell. The overall shape of the curves is very similar, and according to the results of the KS-test (Table 1, values above main diagonal) there were no significant differences on the $1 \%$ level between the single individuals $(1 \mathrm{n}-4 \mathrm{n})$, who were randomly selected.

Analysis of radial positions of CT8 in corresponding carcinomas from five patients (Fig. 2B) revealed a peripheral localization of CT8 close to the nuclear border, comparable to the normal epithelium. In effect, four of the five curves show characteristics rather similar to those of the normal group. They have their maximum of $8.5 \%$ to $10.7 \%$ of relative DNA-content at $68 \%$ to $81 \%$ of the relative nuclear radius. Only one curve (patient 1 in Fig. 2B) shows two maxima at 50\% (7\% relative DNA-content) and $84 \%$ (6.2\% relative DNA-content) of the relative radius with a minimum at $70 \%$ ( $2.5 \%$ relative DNA-content) of the relative radius in between. It is therefore much more extended than the other radial DNA distributions. Comparing all five curves of the carcinoma specimens (Fig. 2B) they show more variability than the set of curves of the normal group (Fig. 2A). According to the KS-test, however, there are no significant differences between the radial DNA distributions of the different tissue samples (1\% level, Table 1). 

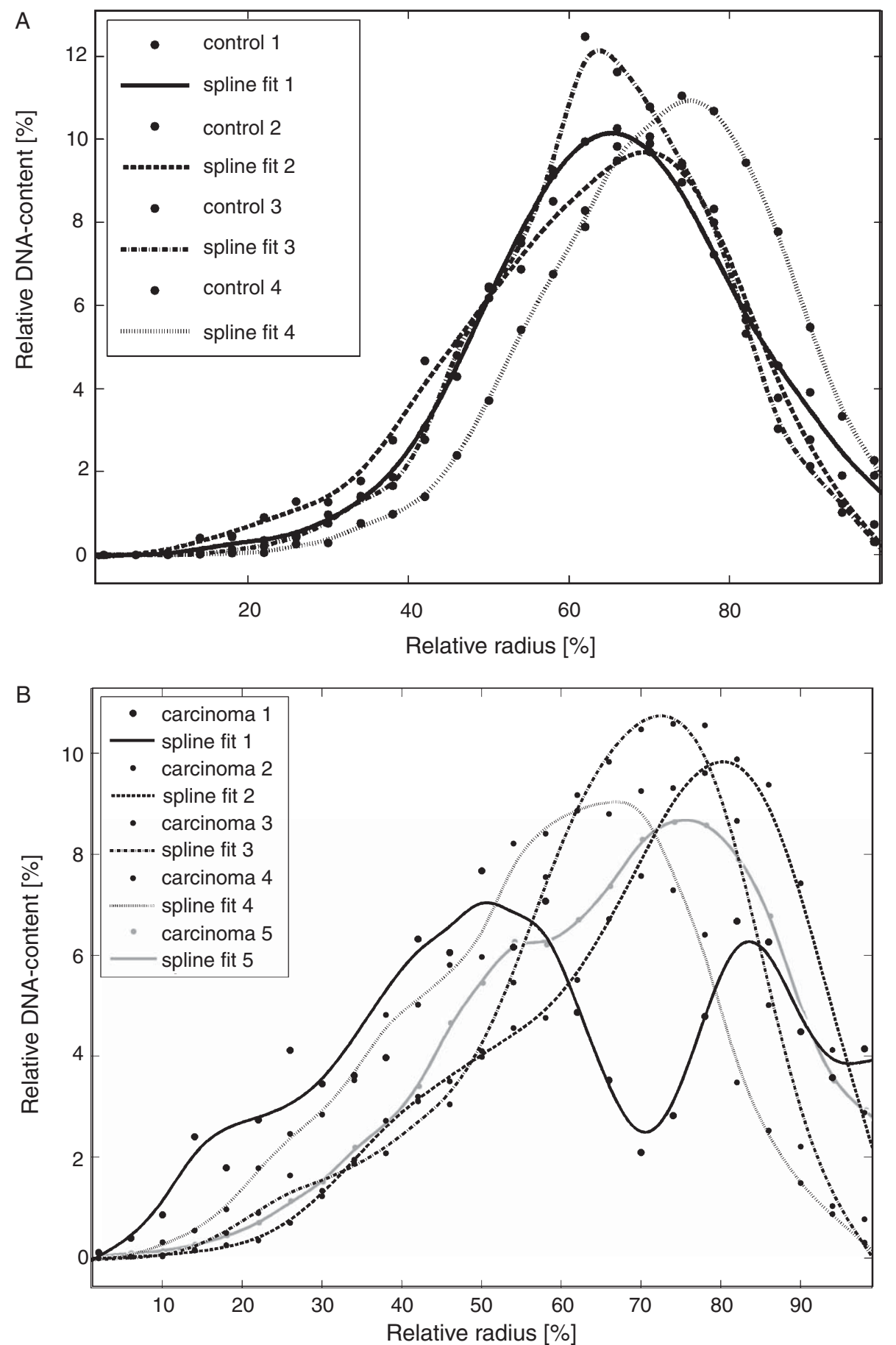

Fig. 2. A: Relative DNA-content within 25 normalized shells of the nucleus for all four normal tissues. In addition to the measured values, a smoothing spline is displayed for each patient data set. B: Relative DNA-content in 25 normalized shells of the nucleus for all five carcinoma tissues. In addition to the measured values, a smoothing spline is displayed for each patient data set. Note the increased variability of the curves as compared to the normal tissues. 
Table 1

Statistical significance test ${ }^{\mathrm{a}}$

\begin{tabular}{lccccccccccccccc}
\hline No. & $1 \mathrm{n}$ & $2 \mathrm{n}$ & $3 \mathrm{n}$ & $4 \mathrm{n}$ & $1 \mathrm{c}$ & $2 \mathrm{c}$ & $3 \mathrm{c}$ & $4 \mathrm{c}$ & $5 \mathrm{c}$ & 6 & 7 \\
\hline $1 \mathrm{n}$ & - & 0.056 & 0.056 & 0.166 & 0.242 & 0.188 & 0.057 & 0.154 & 0.095 & 0.044 & 0.056 & 0.030 & 0.091 \\
$2 \mathrm{n}$ & 0.609 & - & 0.062 & 0.185 & 0.187 & 0.207 & 0.086 & 0.110 & 0.118 & 0.066 & 0.080 & 0.068 & 0.079 \\
$3 \mathrm{n}$ & 0.303 & 0.913 & - & 0.203 & 0.248 & 0.229 & 0.091 & 0.161 & 0.140 & 0.088 & 0.085 & 0.067 & 0.099 \\
$4 \mathrm{n}$ & 1.362 & 1.597 & 1.437 & - & 0.346 & 0.083 & 0.112 & 0.295 & 0.132 & 0.136 & 0.122 & 0.136 & 0.182 \\
$1 \mathrm{c}$ & 3.780 & 3.780 & 3.780 & 3.780 & - & 0.292 & 0.255 & 0.165 & 0.220 & 0.213 & 0.243 & 0.253 & 0.165 \\
2c & 3.782 & 3.780 & 3.780 & 3.780 & 1.814 & - & 0.160 & 0.302 & 0.097 & 0.144 & 0.169 & 0.166 & 0.148 \\
$3 \mathrm{c}$ & 3.782 & 3.801 & 3.780 & 3.780 & 0.529 & 1.853 & - & 0.190 & 0.089 & 0.043 & 0.014 & 0.033 & 0.094 \\
$4 \mathrm{c}$ & 3.835 & 3.854 & 3.833 & 3.833 & 3.832 & 1.073 & 2.529 & - & 0.206 & 0.159 & 0.190 & 0.172 & 0.155 \\
$5 \mathrm{c}$ & 3.782 & 3.801 & 3.781 & 3.781 & 2.609 & 0.832 & 2.192 & 0.996 & - & 0.055 & 0.092 & 0.077 & 0.059 \\
6 & 1.971 & 1.980 & 2.160 & 2.495 & 3.787 & 3.787 & 3.560 & 3.780 & 3.628 & - & 0.039 & 0.044 & 0.052 \\
7 & 0.682 & 1.142 & 0.910 & 1.814 & 3.785 & 3.785 & 3.785 & 3.780 & 3.780 & 1.669 & - & 0.034 & 0.080 \\
$8 \mathrm{n}$ & 0.151 & 0.684 & 0.605 & 1.966 & 3.780 & 3.780 & 3.780 & 3.832 & 3.780 & 1.742 & 0.227 \\
$9 \mathrm{c}$ & 3.782 & 3.801 & 3.781 & 3.781 & 1.285 & 0.907 & 1.436 & 2.759 & 1.965 & 3.787 & 3.785 & 3.780 & 0.094 \\
\hline
\end{tabular}

${ }^{a}$ Above main diagonal: Critical values $\mathrm{d}_{\max }$ of the KS-Test for pairs of radial DNA distributions of different tissues. Values above 0.317 (0.288) show differences at the $1 \%(2.5 \%)$ significance level. Below main diagonal: Z-values of the Mann-Whitney U-Test for pairs of roundness values of different tissues. They define the difference in multiples of standard deviations of the standardized normal distribution $\mathrm{N}(0,1)$. $1 \mathrm{n}-4 \mathrm{n}$ : Normal tissue; 1c-5c: Carcinoma tissue; 6, 7: Test patient; 8n: Cumulative radial distribution of 1n-4n. 9c: Cumulative radial distribution of 1c-5c.

Comparing the results for CT8 positioning in normal ducts with those in neoplastic ducts (carcinoma) does not reveal significant differences of the radial CT8 position distributions on the $1 \%$ level (Table 1) with one exception: The radial distribution of patient 1 (carcinoma 1c) is significantly different from the control specimen of patient 4 (4n). In fact, the carcinoma tissue CT8 distribution of patient 4 (4c) is also different from its corresponding normal tissue $4 \mathrm{n}$ and from the carcinoma CT8 distribution of patient 2 (2c) on the $2.5 \%$ level. This is also fulfilled for patient 1 (1c) in comparison to patient 2 (2c). Still, the cumulative curves of all patients of the respective distributions (Fig. 3) show obviously different characteristics. The maximum of the cumulative relative DNA-content is shifted from $10.5 \%$ at relative radius $68 \%$ for the cumulative normal group to $7.5 \%$ at $75 \%$ relative radius for the carcinoma group. There is also a faster increase of the curve in the carcinoma distribution for small relative radii compared to the distribution of the normal group which resembles a Gaussian distribution.

\subsection{Standardized volume of CT8}

The standardized CT8 volume is dependent on the threshold chosen, because voxels with low chromatin content are excluded when a high threshold is used for image segmentation. Therefore, volume determination is not a very reliable process and absolute values are difficult to be determined. They showed differences in normal pancreatic duct epithelium for each individual and a statistical comparison using the Mann-Whitney U-Test detected significant variation between several samples of the CT8 in carcinoma cells. Regarding the results of standardized CT8 volumes in carcinoma, there was a difference between the individuals as well, as the statistical evaluation with the U-Test demonstrated (data not shown). However, when comparing the standardized CT8 volume in normal epithelium and carcinoma of one individual using the U-Test there was no statistical difference.

This shows that, on the basis of threshold dependent volume detection, a reliable method using absolute values of volumes of CT8 cannot be presented at the moment.

\subsection{Standardized surface of CT8}

The results of the standardized surface of CT8 were similar to those of the volume. The same methodical problems occurred when automatic detection of the surface voxels was attempted. Using the threshold dependent surface functions as a basis for the U-test, we achieved similar results as in the volume case. Individuals showed different standardized CT8 surfaces in normal epithelium, as well as in carcinoma. Statistical evaluation with the U-Test demonstrates significant variation of standardized CT8 surfaces between the individuals (data not shown). Comparing the values of the CT8 surface related to the malignant transfor- 


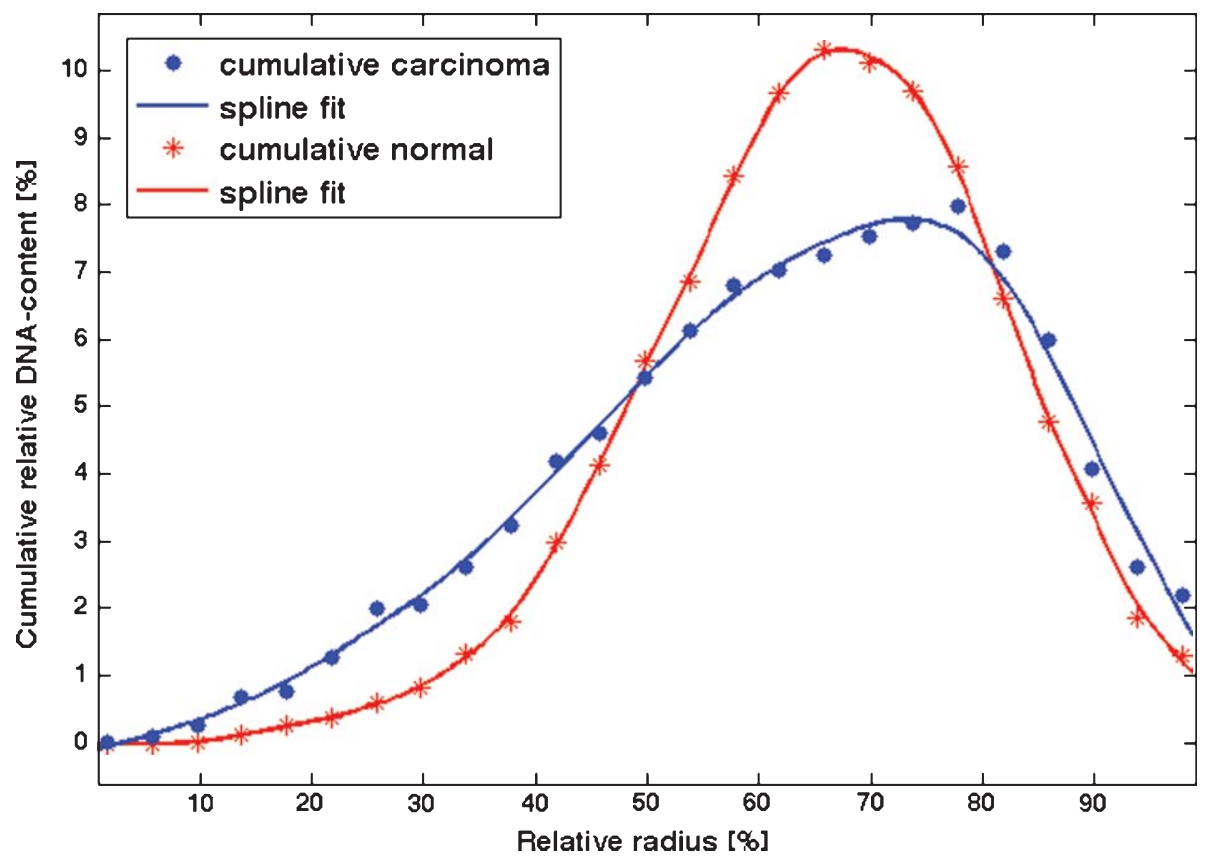

Fig. 3. Cumulative relative DNA-content within 25 normalized shells of the nucleus. The curve of carcinoma tissue consists of the average of 5 patients, the normal curve of the average of 4 patients.

mation, no significant differences were seen between normal and neoplastic (carcinoma) epithelium of one individual. Therefore, absolute surface measurements were not taken into consideration, either.

\subsection{Standardized roundness of CT8}

A solution to the problem of achieving reliable geometry related measurements seems to be the use of the roundness value. In fact, by relating the volume to the surface in a dimensionless way by using appropriate powers of these measurements, the effect of the threshold is mostly eliminated. Therefore, we determined the roundness value using the measurements of volume and surface in the threshold dependent manner as described above and computed the roundness quotient for each threshold separately. This curve is still threshold dependent, but in the ideal case, when the threshold is increased, the effect on volume and surface voxel determination should cancel out in the quotient and the resulting value of the roundness should be constant. This is not the case exactly, probably due to scaling properties of the volume voxels and their surfaces, but there is a linear part in the plot with a rather low factor. To compute a roundness value of the CT8, we used this part of the plot.

Table 2 shows the roundness values for all 13 investigated CT8 objects, including the cumulative cases.

Table 2

Roundness values of the linear part of the roundness function ${ }^{\mathrm{a}}$

\begin{tabular}{lcc}
\hline No. & Roundness & \pm st.dev. \\
\hline $1 \mathrm{n}$ & 0.621 & 0.052 \\
$\mathrm{nn}$ & 0.617 & 0.008 \\
$\mathrm{3n}$ & 0.616 & 0.028 \\
$4 \mathrm{n}$ & 0.636 & 0.034 \\
$1 \mathrm{c}$ & 0.341 & 0.065 \\
$\mathrm{2c}$ & 0.415 & 0.078 \\
$3 \mathrm{c}$ & 0.338 & 0.103 \\
$4 \mathrm{c}$ & 0.457 & 0.014 \\
$5 \mathrm{c}$ & 0.436 & 0.062 \\
6 & 0.581 & 0.048 \\
7 & 0.606 & 0.041 \\
$8 \mathrm{n}$ & 0.609 & 0.023 \\
$9 \mathrm{c}$ & 0.380 & 0.019 \\
\hline
\end{tabular}

${ }^{a}$ Roundness values and their standard deviation as determined in the linear part of the threshold/roundness function. $1 \mathrm{n}-4 \mathrm{n}$ : Normal tissue; 1c-5c: Carcinoma tissue; 6, 7: Test patient; 8n: Cumulative radial distribution of $1 \mathrm{n}-4 \mathrm{n}$; $9 \mathrm{c}$ : Cumulative radial distribution of $1 \mathrm{c}-5 \mathrm{c}$. 
In Table 1, the values below the main diagonal show the values of the U-test for all possible pairs, applied to the linear part of the roundness/threshold functions.

At thresholds between 140 and 210 the standardized roundness of CT8 in normal pancreatic epithelium (numbers $1 \mathrm{n}$ through $4 \mathrm{n}$ ) contains a linear part between the linear fit thresholds (LFT) chosen (see Section 2.7), which results in mean values of about 0.6 , indicating that CT8 was rather round. There were no statistically significant differences (Mann-Whitney U-test: all values below 2 standard deviations) between the CT8 roundness between the individuals $1 \mathrm{n}-4 \mathrm{n}$ (Table 1, below main diagonal), showing a quite homogenous pattern.

Compared to the group of normal epithelium, CT8 seemed to be markedly deformed after malignant transformation (cases $1 \mathrm{c}-5 \mathrm{c}$ ). The values at intermediate thresholds were at 0.2 to 0.4 , being far from the value 1 for the roundest object, the sphere. Additionally, the linear dependence of the normalized roundness and the threshold in the linear fit region is different, as is exemplified by the data for patient 1 (Fig. 4). The linear factor for the carcinoma tissue is greater than for the normal tissue. The same can be seen in Fig. 5, which shows the cumulative roundness for both tissue types. The roundness values for carcinoma cases do not show significant inter-individual differences (Table 1).

Statistical comparison of normal $(1 n-4 n)$ cells versus carcinoma $(1 \mathrm{c}-4 \mathrm{c})$ cells of one individual using the U-Test reveal significant differences of standardized roundness of CT8 (Mann-Whitney U-test: all values above 3 standard deviations; Table 1). This indicates a decrease of CT8 roundness in pancreatic epithelium during malignant transformation.

\subsection{Analysis and classification of the test patient}

As experimental conditions can have a considerable influence on the outcome of image quality and subsequent microscopic analysis, we investigated the tissue of one patient several times and compared the results to the other specimens (numbers 6 and 7, Tables 1 and 2). According to the linear fit of the roundness values the patient's tissue was classified correctly as normal (Fig. 5).

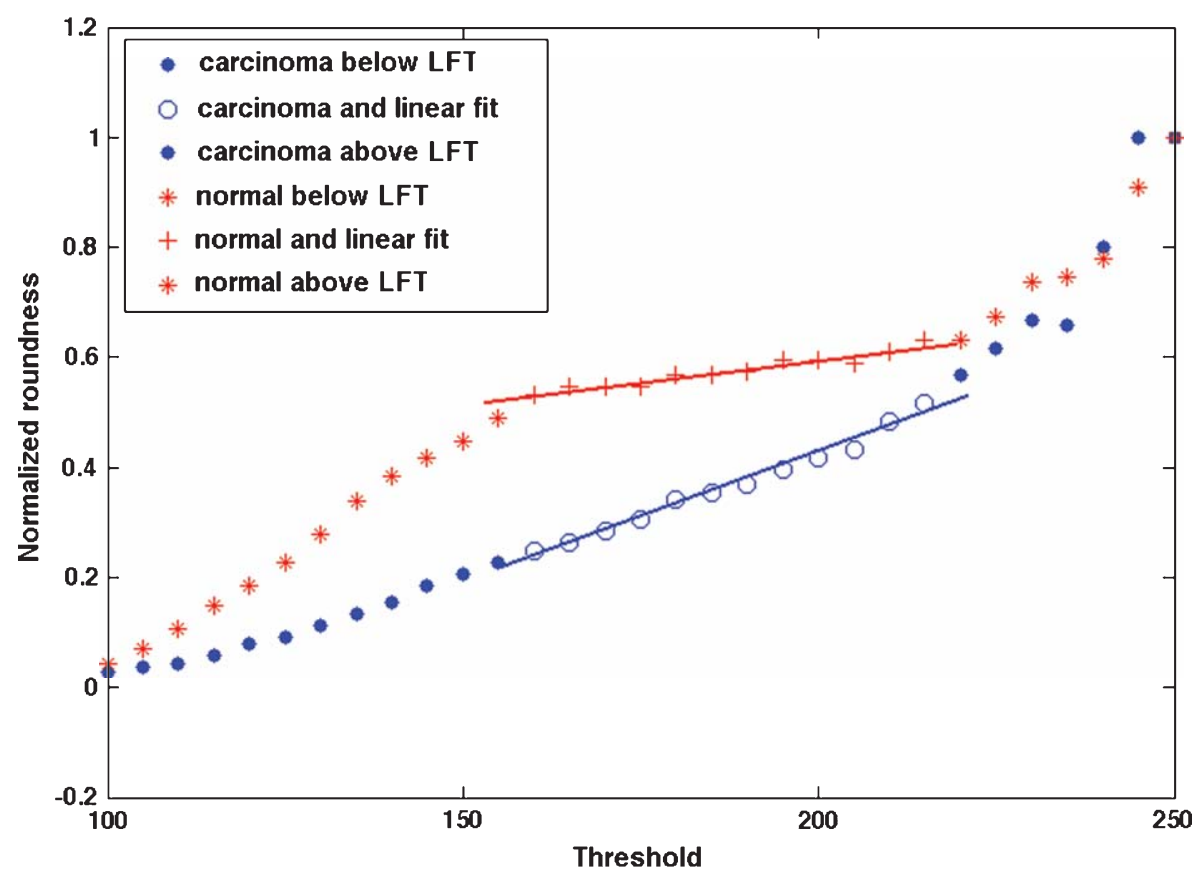

Fig. 4. Relative roundness in dependence of the intensity threshold for carcinoma and normal tissue of patient 1 . The linear fits shown have been calculated for the values between threshold (LFT) 170 and 230. The mean roundness values based on this linear fit for carcinoma and normal tissue are significantly different. 


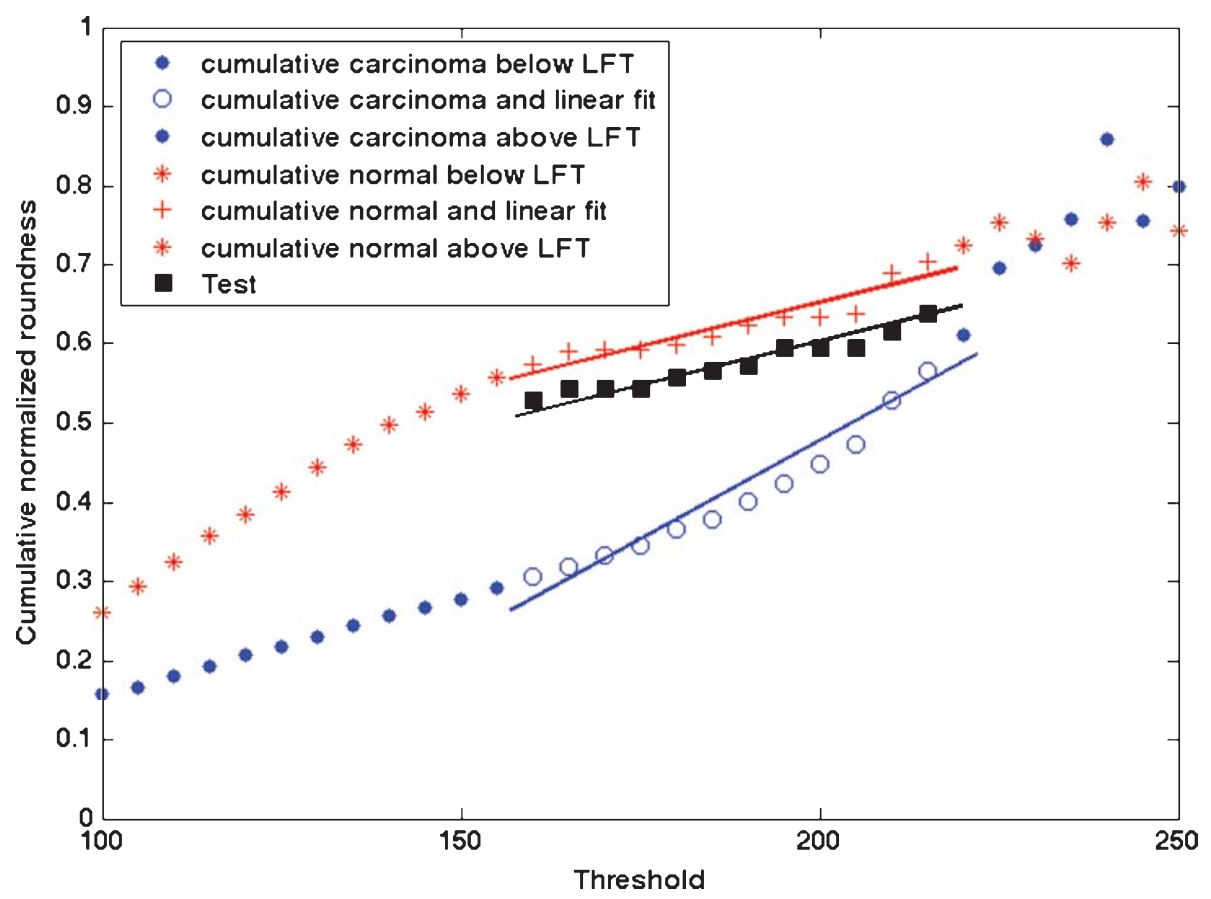

Fig. 5. Cumulative relative roundness in dependence of the intensity threshold. The curve of carcinoma tissue consists of the average of 5 patients, the normal curve of the average of 4 patients. The linear fits shown have been calculated for the values between threshold (LFT) 170 and 230. The mean roundness values based on this linear fit for carcinoma and normal tissue are significantly different. In addition, the values for the test patient are shown and clearly demonstrate that the specimen can be classified as normal.

\section{Discussion}

In this study we investigated the radial positioning, the volume, the surface, and the roundness of CT8 in nuclei of non-neoplastic (normal) pancreatic ductal epithelium in comparison to neoplastic pancreatic ductal adenocarcinoma as a model of malignant transformation. We evaluated these parameters while conserving the histological context by using $15 \mu \mathrm{m}$ sections from formalin-fixed, paraffin-wax embedded tissues. A lot of published studies analyzing nuclear and chromosome architecture used fixed cells of in vitro cell cultures with advantages, such as high uniformity of the cells and without suspected structural artificial changes when using formalin-fixed paraffin wax-embedded tissue. On the other hand, it is also well known that in vitro cells from cell cultures exhibit a different architecture than cells remaining in the microanatomical context [24]. Interestingly, the results of CT8 architecture described in tissue sections here did not differ basically from the results of cell cultures. For example, the CT8 radial position in peripheral lymphoblasts was also found at the nuclear periphery [5]. In computer simulated models of morphologically preserved lymphocytes similar values were also found for radial positioning, volume, and roundness [22]. Therefore, paraffin-wax embedded tissue seems to be a valuable source for investigation of genome architecture [45] to complement studies using in vitro cultured cell lines.

The most important methodological step is FISH and its pretreatment. Chemical and thermal denaturation procedures are necessary to provide for the accessibility of the WCP probe specifically to its complementary target. These procedures of course may be destructive for chromatin and genome organization to a certain degree. Moreover, the biophysical properties of normal and neoplastic cells may be different, so that we can not completely exclude the possibility of the FISH pretreatment protocol affecting the results of shape parameters as a biased error. The results presented here, however, suggest that the different changes are patient independent, so that the different roundness val- 
ues between normal and neoplastic cells at least seem to be a recurrent finding.

It has been well established that in cell nuclei each CT takes a certain tissue specific intra-nuclear position [31] which is known to be non-random [10, 26, 29]. From these results, however, it remained unclear whether there are variations between individual samples. Our analyses of over 100 randomly selected non-neoplastic nuclei of pancreatic duct epithelium were therefore taken from different patients. The data revealed a localization of CT8 towards the nuclear periphery, which was independent from the individual being analyzed. As previously suggested [45], CT8 showed a slightly shifted peripheral position in pancreatic ductal adenocarcinoma accompanied by a higher individual dependent variability. Although CT8 contains tumor relevant gene domains as, for instance, CMYC the peripheral shift towards the border was not statistically significant compared to normal epithelium. This observation is concordant with studies that describe a peripheral location of chromosomes with a low gene density and activity $[13,15]$ and with investigations reporting no significant changes of radial positioning of CTs during malignant transformation in cell lines [10].

On the other hand, no significant change of the relative radial CT8 position during the malignant transformation is surprising, since radial positioning of CTs and sub-chromosomal elements are main features correlated to genome function [42] or dysfunction [46]. However, assuming that genome function and molecular accessibility of gene domains are correlated, changes of the CT shape may reflect an improved accessibility of cancer related genes. Thus, the slight shift of the CT position may be a consequence of the shape modifications. Moreover, modifications of other chromosome shapes associated with genomic alterations in cancer may contribute to the higher individual variation of CT positions in general.

Normalized volumes and surfaces of CT8 may differ between individuals. Nevertheless, there were no significant differences between normal (non-neoplastic) and carcinoma (neoplastic) epithelium from the same individual. In light microscopy an increased nuclear volume of malignant cells compared to normal cells can be observed. One might expect a parallel increase of the CT volumes and surface in malignant nuclei due to chromatin disaggregation by a higher transcription rate or DNA amplifications. Considering all methodical shortcomings described in results, it appears that this phenomenon was found for the absolute values but not for the normalized values. Thus, there only seems to be a proportional increase of CT volume and surface.

Interestingly, although no statistically significant differences of normalized volume and surface were found, the results of the normalized CT roundness showed striking differences. Across all individuals the roundness of CT8 in normal epithelium is quite spheroidal. In contrast, in carcinoma epithelium CT8 seems to be shaped more irregularly. These results confirm our previous observation of a decreased roundness in carcinoma cells [45] that is comparable with a decreased roundness of nuclei of malignant cells, as already visible in $\mathrm{H} \& \mathrm{E}$ stained sections by light microscopy. Thus, spatial changes of CT during malignant transformation, like increased absolute volume and surface, as well as decreased normalized roundness seem to mimic changes of the nuclei themselves.

Of course, there is still a huge gap to be bridged between topological/architectural genomic findings and functional data obtained by the spectrum of molecular biological techniques. Basic requirement for discussing a relevant association of structural and functional data is the reliability of the results. As pointed out above, a critical methodological step in the investigation of genome architectural parameters using paraffin-embedded tissue is the FISH pretreatment procedure, so that we cannot completely exclude a biased distortion of the territories in neoplastic cells due to a higher vulnerability to pretreatment compared to normal cells.

Provided that our observation reflects a real difference, one might suggest that the loss of roundness of CT8 accompanies the malignant transformation, analogous to shape changes of nuclei of preinvasive dysplastic cells. It is discussed that an altered chromosomal architecture affects the regular gene expression by, for instance, disturbing the docking of transcription factors on promoter or enhancer sequences [16]. Primary genomic changes during malignant transformation may cause spatial changes of the CT architecture. Since for example the oncogene $C M Y C$ is amplified in nearly $30 \%$ of adenocarcinoma of the pancreas, genomic amplifications or losses may impair the chromosomal architecture and lead to a decreased CT8 roundness. Besides, there are some genes like $S A T B 1, C T C F$ and $S / M A R$ coding proteins that seem to keep up the nuclear architecture [27]. A modification of these genes leads to heavy interferences of the 
regular nuclear architecture followed by a disturbed expression of other genes. There might be an early genetic or epigenetic event leading to a chromosomal deformation that in turn causes genomic instability. This, however, remains speculative and should be subject of a more detailed analysis of the nuclear and chromosomal architecture, which may require more advanced labeling and imaging technologies. Recent technological improvements like COMBO-FISH [18] for specific labeling of small genome targets and high resolution localization microscopy (e.g. [25]) may improve microscopic imaging to obtain more detailed information about the micro- and nano-architecture of genomes. Grid computing resources offer in addition new possibilities for a complex analysis of individual dependent changes of multidimensional architectural parameters [37, 38].

In conclusion, the results presented here show the usefulness of archival tissue materials. The data indicate a deformation of the interphase chromosome 8 territory in pancreatic ductal adenocarcinoma. However, whether this really is associated with genomic instability in tumor cells remains to be elucidated by means of novel technologies. Moreover, further studies could investigate preinvasive lesions in order to determine the time course of changing functionally correlated architectural parameters during malignant transformation.

\section{Acknowledgements}

Funding of the German Ministry of Education and Research (BMBF) (FKZ 13N8350 and FKZ 01IG07015 G - "Services@MediGRID”) to Michael Hausmann is gratefully acknowledged. We thank Mrs. Liselotte Bokla for excellent technical assistance. Our special thanks go to Dr. A.L. Ibaba, IDISCC (International Desert Institute for Scientific Cloud Computing, Beer Sheva, Israel) for stimulating discussions and information about parallel lustre data systems and Grid capacities.

\section{References}

[1] D.G. Altmann, Practical Statistics For Medical Research, Chapman and Hall, London, 1991, pp. 194-197.

[2] E. Bártová, S. Kozubek, P. Jirsová, M. Kozubek, H. Gajová, E. Lukásová, M. Skalniková, A. Ganová, I. Koutná and M. Hausmann, Nuclear topography and gene activity in human differentiated cells, J Struct Biol 139 (2002), 76-89.
[3] A. Bolzer, G. Kreth, I. Solovei, D. Koehler, K. Saracoglu, C. Fauth, S. Müller, R. Eils, C. Cremer, M.R. Speicher and T. Cremer, Three-dimensional maps of all chromosomes in human male fibroblast nuclei and prometaphase rosettes, $P L O S$ Biol 3 (2005), e157.

[4] T. Boveri, Die Blastomerenkerne von ascaris megalocephala und die Theorie der Chromosomenindividualität, Archiv für Zellforschung 3 (1909), 181-268.

[5] S. Boyle, S. Gilchrist, J.M. Bridger, N.L. Mahy, J.A. Ellis and W.A. Bickmore, The spatial organization of human chromosomes within the nuclei of normal and emerin-mutant cells, Hum Mol Genet 10 (2001), 211-219.

[6] T. Cremer and C. Cremer, Chromosome territories, nuclear architecture and gene regulation in mammalian cells, Nat Rev Genet 2 (2001), 292-301.

[7] T. Cremer and C. Cremer, Rise, fall and resurrection of chromosome territories: a historical perspective. Part I. The rise of chromosome territories, Eur J Histochem 50 (2006), 161-176.

[8] T. Cremer and C. Cremer, Rise, fall and resurrection of chromosome territories: a historical perspective. Part II. Fall and resurrection of chromosome territories during the 1950s to 1980s. Part III. Chromosome territories and the functional nuclear architecture: experiments and models from the 1990s to the present, Eur J Histochem 50 (2006), 223-272.

[9] T. Cremer, A. Kurz, R. Zirbel, S. Dietzel, B. Rinke, E. Schröck, M.R. Speicher, U. Mathieu, A. Jauch, H. Emmerich, H. Scherthan, T. Ried, C. Cremer and P. Lichter, Role of chromosome terriories in the functional compartmentalization of the cell nucleus, Cold Spring Harb Symp Quant Biol 58 (1993), 777-792.

[10] M. Cremer, K. Küpper, B. Wagler, L. Wizelman, J. von Hase, Y. Weiland, L. Kreja, J. Diebold, M.R. Speicher and T. Cremer, Inheritance of gene density-related higher order chromatin arrangements in normal and tumor cell nuclei, $J$ Cell Biol 162 (2003), 809-820.

[11] T. Cremer, K. Kupper, S. Dietzel and S. Fakan, Higher order chromatin architecture in the cell nucleus: on the way from structure to function, Biol Cell 96 (2004), 555-567.

[12] T. Cremer, M. Cremer, S. Dietzel, S. Müller, I. Solovei and S. Fakan, Chromosome territories - a functional nuclear landscape, Curr Opin Cell Biol 18 (2006), 307-316.

[13] J.A. Croft, J.M. Bridger, S. Boyle, P. Perry, P. Teague and W.A. Bickmore, Differences in the localization and morphology of chromosomes in the human nucleus, J Cell Biol 145 (1999), 1119-1131.

[14] R. van Driel, P.F. Fransz and P.J. Verschure, The eukaryotic genome: a system regulated at different hierarchical levels, J Cell Sci 116 (2003), 4067-4075.

[15] M. Falk, E. Lucasova, S. Kozubek and M. Kozubek, Topography of genetic elements of X-chromosome relative to the cell nucleus and to the chromosome $\mathrm{X}$ territory determined for human lymphocytes, Gene 292 (2002), 13-24.

[16] P. Fraser and W. Brickmore, Nuclear organization of the genome and the potential for gene regulation, Nature 447 (2007), 413-417.

[17] S.R. Hamilton and L.A. Aaltonen, Pathology and Genetics of Tumours of the Digestive System WHO Classification of Tumours, IARC Press, Lyon, 2000, pp. 219-250.

[18] M. Hausmann, G. Hildenbrand, J. Schwarz-Finsterle, U. Spöri, H. Schneider, E. Schmitt and C. Cremer, New technologies 
measure genome domains - high resolution microscopy and novel labeling procedures enable 3-D studies of the functional architecture of gene domains in cell nuclei, Biophotonics Int 12(10) (2005), 34-37.

[19] S.H. Kim, P.G. McQueen, M.K. Lichtman, E.M. Shevach, L.A. Parada and T. Misteli, Spatial genome organization during T-cell differentiation, Cytogenet Genome Res 105 (2004), 292-301.

[20] S. Kozubek, E. Lukasova, P. Jirsova, I. Koutna, M. Kozubek, A. Ganova, E. Bartová, M. Falk and R. Pasekova, 3D Structure of the human genome: order in randomness, Chromosoma 111 (2002), 321-331.

[21] D.E Knuth, The Art of Computer Programming, Addison Reading, UK.

[22] G. Kreth, J. Finsterle, J. von Hase, M. Cremer and C. Cremer, Radial arrangement of chromosome territories in human cell nuclei: a computer model approach based on gene density indicates a probabilistic global positioning code, Biophys $J \mathbf{8 6}$ (2004), 2803-2812.

[23] C. Lanctôt, T. Cheutin, M. Cremer, G. Cavalli and T. Cremer, Dynamic genome architecture in the nuclear space: regulation of gene expression in three dimensions, Nat Rev Genet 8 (2007), 104-115.

[24] S.A. Lelievre, V.M. Weaver, J.A. Nickerson, C.A. Larabell, A Bhaumik, O.W. Petersen and M.J. Bissell, Tissue phenotype depends on reciprocal interactions between the extracellular matrix and the structural organization of the nucleus, Proc Natl Acad Sci U S A 95 (1998), 14711-14716.

[25] P. Lemmer, M. Gunkel, Y. Weiland, P. Müller, D. Baddeley, R. Kaufmann, A. Urich, H. Eipel, R. Amberger, M. Hausmann and C. Cremer, Using conventional fluorescent markers for farfield fluorescence localization nanoscopy allows resolution in the $10 \mathrm{~nm}$ range, J Microsc 235 (2009), 163-171.

[26] E. Lukasova, S. Kozubek, M. Kozubeck, M. Falk and J. Amrichova, The 3D structure of human chromosomes in cell nuclei, Chromosome Res 10 (2002), 535-548.

[27] J. Mateos-Langerak, S. Goetze, H. Leonhardt, T. Cremer, R. van Driel and C. Lanctôt, Nuclear Architecture: Is it important for genome function and can we prove it? J Cell Biochem 102 (2007), 1067-1075.

[28] K.J. Meaburn, P.R. Gudla, S. Khan, S.J. Lockett and T. Misteli, Disease-specific gene repositioning in breast cancer, $J$ Cell Biol 187(6) (2009), 801-812.

[29] T. Misteli, Beyond the sequence: cellular organization of genome function, Cell 128 (2007), 787-800.

[30] L.A. Parada and T. Misteli, Chromosome positioning in the interphase nucleus, Trends Cell Biol 12 (2002), 425-432.

[31] L.A. Parada, P.G. McQueen and T. Misteli, Tissue-specific spatial organization of genomes, Genome Biol 5 (2004), R44.

[32] T. Pederson, The spatial organization of the genome in mammalian cells, Curr Opin Genet Dev 14 (2004), 203-209.

[33] A. Pombo and M.R. Branco, Functional organisation of the genome during interphase, Curr Opin Genet Dev 17 (2007), $1-5$.
[34] H. Reinsch, Smoothing by spline functions, Numerische Mathematik 10 (1967), 177-183.

[35] J. Rouquette, C. Cremer, T. Cremer and S. Fakan, Functional nuclear architecture studied by microscopy: present and future, Int Rev Cell Mol Biol 281 (2010), 1-90.

[36] C. Schleger, C. Verbeke, R. Hildenbrand, H. Zentgraf and U. Bleyl, c-MYC activation in primary and metastatic ductal adenocarcinoma of the pancreas: incidence, mechanismus, and clinical significance, Mod Pathol 15 (2002), 462469.

[37] E. Schmitt, S. Stein and M. Hausmann, Conception of an image data base for cell nuclei and geometric algorithms for diagnosis and therapy monitoring, Stud Health Technol Inform 147 (2009), 251-256.

[38] E. Schmitt, P. Müller, S. Stein, J. Schwarz-Finsterle and M. Hausmann, Cell nucleus architecture in health and medicine: geometrical descriptors and their use in grid based case studies, Stud Health Technol Inform 159 (2010), 272-276.

[39] L.H. Sobin, M.K. Gospodarowicz and C. Wittekind, TNM Classification of Malignant Tumours, 7th Edition, Wiley\& Sons, New York, 2009.

[40] D.L. Spector, The dynamics of chromosome organization and gene regulation, Annu Rev Biochem 72 (2003), 573-608.

[41] S. Stein, I. Solovei, M. Cremer, R. Zinner, S. Timme, A. Walch, M. Werner, M. Hausmann and C. Cremer, 3D-image analysis of the topological organisation of chromatin in human lymphocytes, fibroblasts and in cancer cells of formalin-fixed, paraffinembedded tissue sections, Chromosome Res 12 (Suppl. 1) (2004), 34.

[42] T. Takizawa, K.J. Meaburn and T. Misteli, The meaning of gene positioning, Cell 135 (2008), 9-13.

[43] H. Tanabe, S. Muller, M. Neusser, J. von Hase, E. Calcagno, M. Cremer, I. Solovei, C. Cremer and T. Cremer, Evolutionary conservation of chromosome territory arrangements in cell nuclei from higher primates, Proc Natl Acad Sci U S A 99 (2002), 4424-4429.

[44] H. Tanabe, K. Kupper, T. Ishida, M. Neusser and H. Mizusawa, Inter- and intra-specific gene-density-correlated radial chromosome territory arrangements are conserved in old world monkeys, Cytogenet Genome Res 108 (2005), 255-261.

[45] T. Wiech, S. Timme, F. Riede, S. Stein, M. Schuricke, C. Cremer, M. Werner, M. Hausmann and A. Walch, Human archival tissues provide a valuable source for the analysis of spatial genome organization, Histochem Cell Biol 123 (2005), 229-238.

[46] T. Wiech, S. Stein, V. Lachenmaier, E. Schmitt, J. SchwarzFinsterle, E. Wiech, G. Hildenbrand, M. Werner and M. Hausmann, Spatial allelic imbalance of BCL2 genes and chromosome 18 territories in nonneoplastic and neoplastic cervical squamous epithelium, Eur Biophys J 38 (2009), 793-806. 


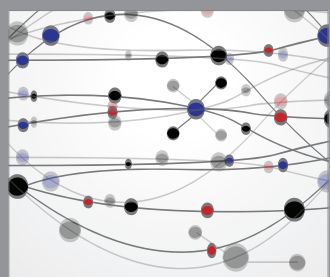

The Scientific World Journal
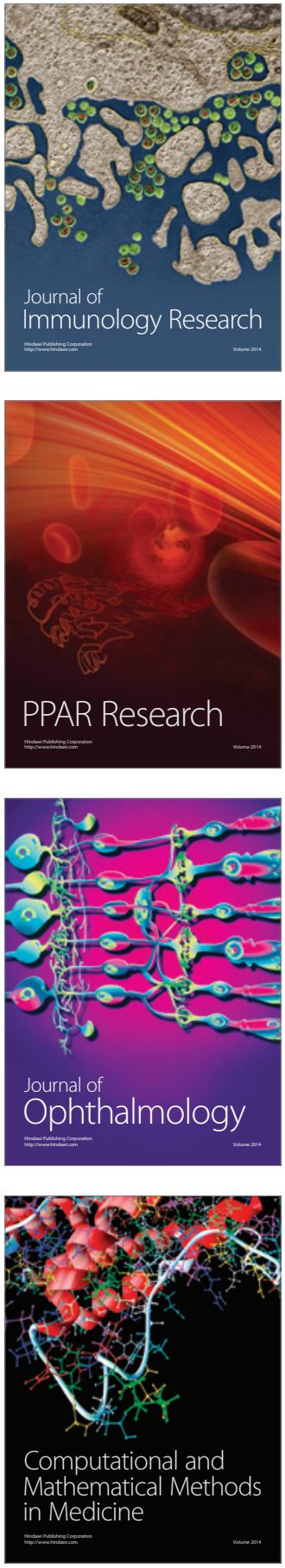

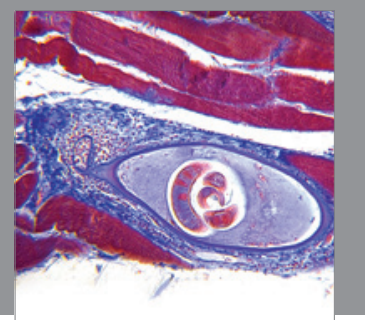

Gastroenterology

Research and Practice
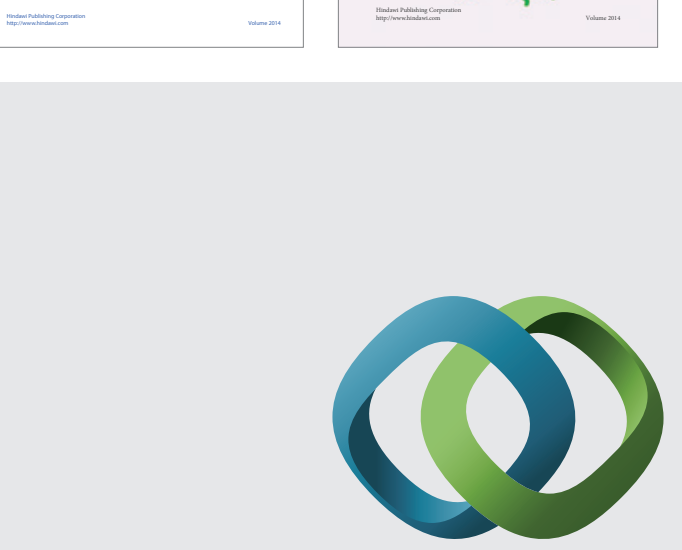

\section{Hindawi}

Submit your manuscripts at

http://www.hindawi.com
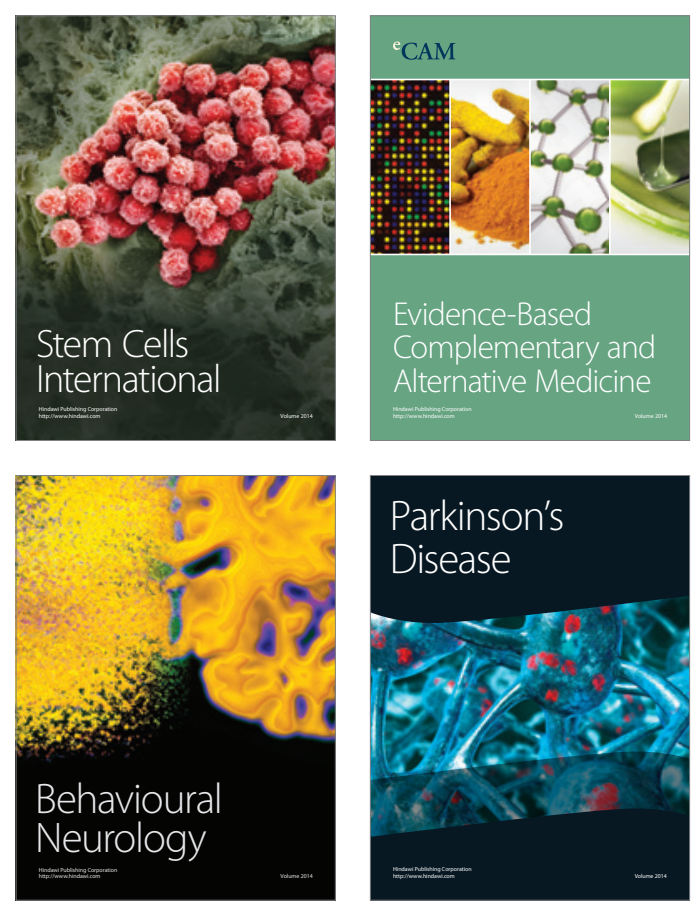

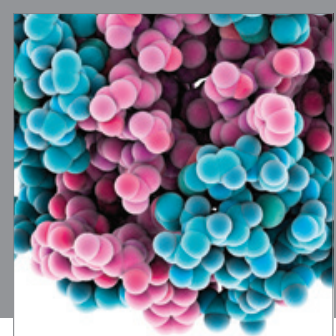

Journal of
Diabetes Research

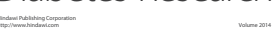

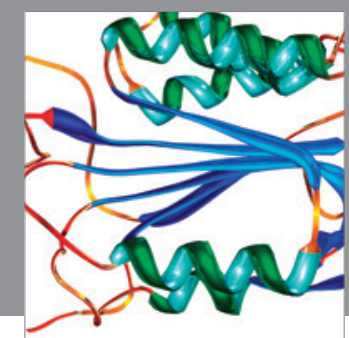

Disease Markers
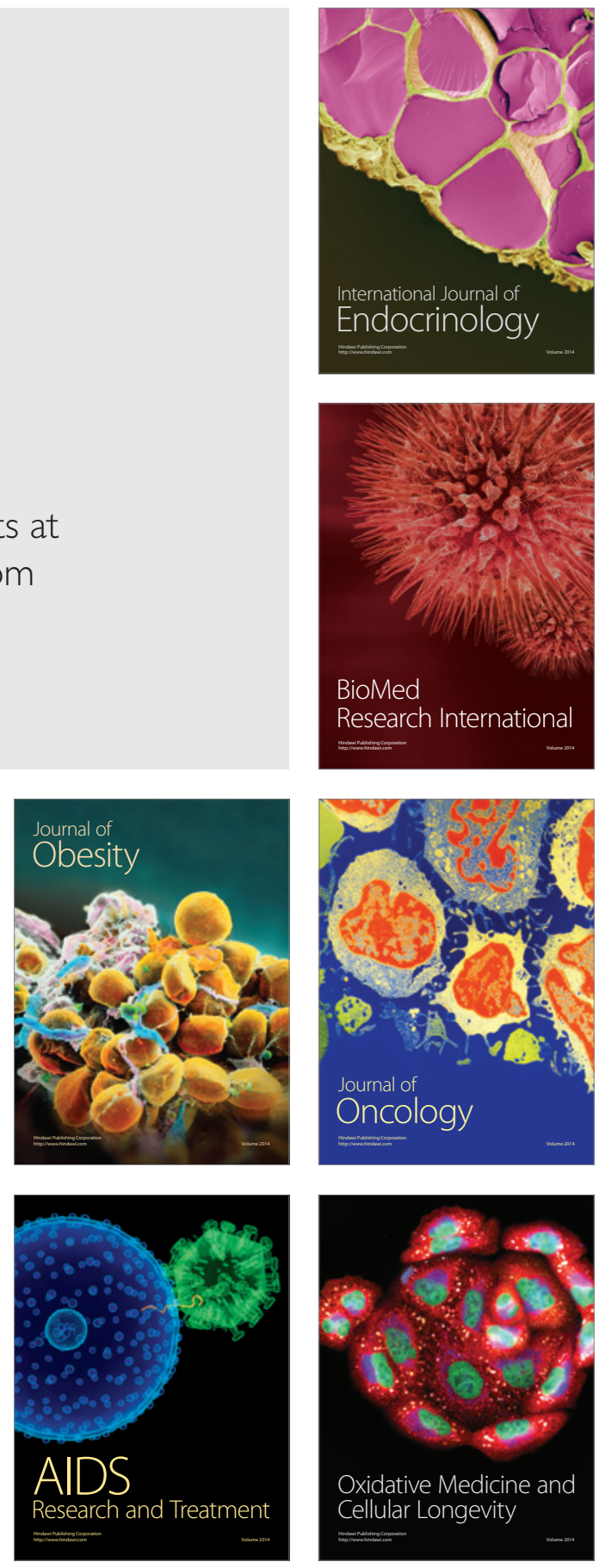